\title{
Multi-objective optimal tuning of two degrees of freedom PID controllers using the ENNC method
}

\author{
Mónica P. Contreras-Leiva ${ }^{1}$, Francisco Rivas ${ }^{1}$, José D. Rojas ${ }^{1}$, Orlando Arrieta ${ }^{1,2}$, \\ Ramon Vilanova ${ }^{3}$, Marian Barbu ${ }^{4}$ \\ ${ }^{1}$ Escuela de Ingeniería Eléctrica, Universidad de Costa Rica, Costa Rica \\ ${ }^{2}$ Instituto de Investigaciones en Ingeniería, Universidad de Costa Rica, Costa Rica \\ ${ }^{3}$ Departament de Telecomunicació i d'Enginyeria de Sistemes, Universitat Autònoma de Barcelona, Spain \\ 4"Dunarea de Jos", University of Galati, Romania \\ email: jdrojas@eie.ucr.ac.cr
}

\begin{abstract}
In this paper the Enhanced Normalized Normal Constrained method is applied to find the optimal tuning of second order PID controllers. This tuning takes into account both the servo and regulatory modes simultaneously, as well as a closed loop robustness criterion. The methodology is successfully applied to a high order linear plant and to a non linear continuous stirred tank reactor model, showing the effectiveness of the optimization methodology to solve control related problems. Also, the method is applied to a benchmark control system that takes into account three different sources of disturbances.
\end{abstract}

Index Terms-PID control, Pareto optimization, Process control, ENNC.

\section{INTRODUCTION}

The design of controlled systems, always have to consider multiple conflicting objectives. With this perspective, the task of the engineer in charge of the control task, becomes to find the optimal compromise between these different objectives [1]-[3].

It is common to tune the parameters of industrial controllers by means of an optimization problem. However, when the control engineer is facing several objectives at once, this optimization problem becomes a multi-objective optimization (MOO) [4]. Particularly for industrial PID controllers, this problem also becomes nonlinear and (possibly) non-convex, which makes it a non-trivial problem.

In this sense, the multi-objective optimization of PID controllers remains an open research topic, even though it has been studied for several decades [5]-[7] and with multiple optimization methods, including bio-inspired techniques such as neural networks, fuzzy logic and genetic algorithms to solve the problem of the optimization [8], [9].

In this paper the Enhanced Normalized Normal Constrained (ENNC) method is used to solve the tuning of a two degrees of freedom (2DoF) PID controller as a multi-objective multivariable non-linear optimization problem.

There exist various methods that transform the multiobjective problem into a single objective optimization by rewriting the problem. The Normalized Normal Constraint (NNC) and Enhanced NNC (ENNC) are two of these methodologies that have been used in a multitude of application 978-1-5090-2720-0/16/\$31.00 @2016 IEEE

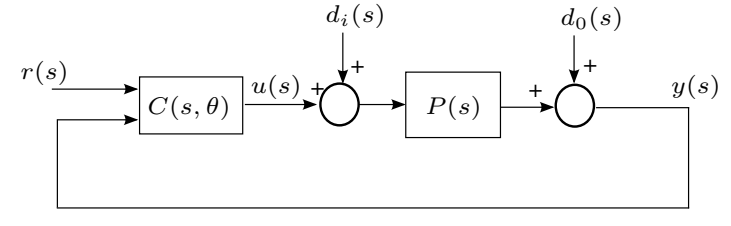

Figure 1. Feedback control loop.

cases. For example, in [7] the optimization of PI controllers for First Order plus time delay (FOPTD) plants is computed using the NNC methods and minimizing the Integral of the Absolute Error (IAE) of the closed-loop response to a change in the reference signal and the Total Variation (TV) of the control signal. In [10], a piecewise NNC method is used to solve a Multi-objective reactive power optimization problem, where the active power loss and the square sum of voltage deviation are simultaneously optimized in an AC-DC hybrid power system.

The contribution of this paper is to apply the ENNC method for the tuning of 2DoF PID controller for high order and nonlinear plants, by means of a linear second order time delayed (SOPTD) models. The IAE is used as the cost function for the reference tracking problem and the input/output disturbance rejection problems simultaneously. In addition, the optimization problem takes into account a measure of the robustness of the closed loop controlled system.

The paper is divided as follows: in Section II, the methodology and the control problem are presented, in Section III, the ENNC method is implemented for the tuning of PID controllers for the control of a high order plant and a non-linear continuous stirred tank reactor (CSTR). Also, the method is applied to the tuning of a PID controller that takes into account change in the reference, input disturbance and output disturbance signals. Conclusions are presented in Section IV.

\section{METHODS}

\section{A. PID control}

A feedback control system as shown in Figure 1, is used as the control topology for the application of the ENNC method in this paper. The elements are: 
- $C(s, \boldsymbol{\theta})$ is the Two Degrees of Freedom (2DoF) Proportional Integral Derivative (PID) controller with parameters $\boldsymbol{\theta}=\left[\begin{array}{cccc}K_{p} & T_{i} & T_{d} & \beta\end{array}\right]^{T}$.

- $P(s)$ represent the controlled process, modeled as a plant of Second-Order Plus Time Delay (SOPTD), with a transfer function of the form:

$$
P(s)=\frac{K e^{-L s}}{\left(T_{1} s+1\right)\left(T_{2} s+1\right)},
$$

It is common to define $T_{2}$ as the less dominant lag time of the system and therefore $T_{2}=a T_{1}$.

The control signal of this controller can be computed as:

$$
u(s)=C_{r}(s, \boldsymbol{\theta}) r(s)-C_{y}(s, \boldsymbol{\theta}) y(s),
$$

where the part applied to the set point signal is called servo controller:

$$
C_{r}(s, \boldsymbol{\theta})=K_{p}\left(\beta+\frac{1}{T_{i} s}\right),
$$

And the controller applied to the feedback signal is call the regulator controller:

$$
C_{y}(s, \boldsymbol{\theta})=K_{p}\left(1+\frac{1}{T_{i} s}+\frac{T_{d} s}{\alpha T_{d} s+1}\right),
$$

$\alpha$ is the constant for the derivative filter.

It is important to guarantee a certain level of robustness for the closed-loop controlled system to ensure that the controlled system has a stable performance against variations in the dynamic of the plant [11], [12]. A measure of the relative stability or robustness, is the maximum sensitivity.

$$
M_{s}=\max _{\omega}\left\{\frac{1}{\left|1+C_{y}(j \omega) P(j \omega)\right|}\right\}
$$

The recommended robustness index value is in the range $1.2 \leq M_{s} \leq 2.0$, where the higher $M_{s}$, the less robust the closed-loop is [6].

\section{B. Control problem}

The controller tuning can be solved as a multi-objective optimization problem. One widely used indicator of performance, is the Integral of Absolute Error (IAE) given by:

$$
J=\int_{0}^{\infty}|e(t)| d t
$$

The error signal $e(t)$ it is calculated with:

$$
e(t)=r(t)-y(t) \text {. }
$$

In the case of disturbance rejection, the error signal becomes:

$$
e_{d}(t)=-y_{d}(t)
$$

Evaluated for a change in the input disturbance $\left(d_{i}\right)$, the error signal becomes:

$$
J_{d i}(\boldsymbol{\theta})=\int_{0}^{\infty}\left|-y_{d i}(t, \boldsymbol{\theta})\right| d t
$$

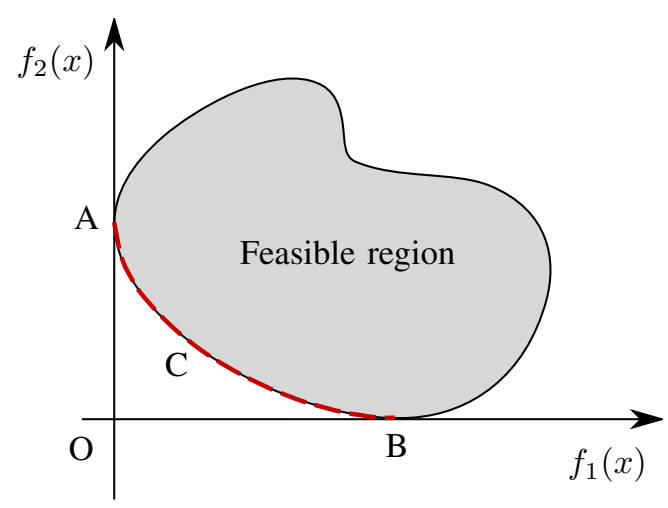

Figure 2. Pareto frontier to optimize two objectives.

where, $y_{d i}$ corresponds to the response to an disturbance input step change. For the change in the reference signal, the criterion is given by:

$$
J_{r}(\boldsymbol{\theta})=\int_{0}^{\infty}\left|r(t)-y_{r}(t, \boldsymbol{\theta})\right| d t,
$$

where $y_{r}$ is the response to a step change in the set-point signal $(r)$. The optimal controller for the regulator response can be obtained by minimizing (9), while the servo response can be optimized by optimizing (10). But usually it is impossible to minimize $J_{d i}$ and $J_{r}$ with the same set of parameters $\boldsymbol{\theta}$ (so this hypothetical solution is known as the utopia point). All solutions that are closest to the utopia point, create the Pareto frontier [13].

For a general explanation of this concept, in Fig. 2 the Pareto front formed with two different cost function $f_{1}(\boldsymbol{x})$ and $f_{2}(\boldsymbol{x})$ corresponds to the arc $A C B$. As it can be seen, when the feasible region (the cost function values that are attainable with the possible allowed values of the decision variables $\boldsymbol{x}$ ) is drawn in a plane whose axis are the values of the objective functions, the Pareto front is the border of the feasible region that gives the minimal values for both objective functions.

The points in the Pareto front can not have lower values in one of the objective function without worsening the other objective function. In this case, the point of utopia would be exactly the origin of the plane (the point $O$ ), because that point represents the minimum value for both cost functions. It should be noted that, in general, this point is not within the feasible region and therefore cannot be achieved. Points A and $\mathrm{B}$ are called anchor points and represents the cases where at least one of the functions are minimal.

Applying these concepts to the control problem and since both functions $\left(J_{d i}\right.$ and $J_{r}$ ) have to be solved at the same time, the controller tuning can be written as a multi-objective optimization problem, by defining the cost function as:

$$
\mathbf{J}(\boldsymbol{\theta})=\left[J_{d i}(\boldsymbol{\theta}), J_{r}(\boldsymbol{\theta})\right]^{T},
$$

and solved by finding all possible optimal solutions of:

$$
\begin{aligned}
& \mathbf{J}\left(\boldsymbol{\theta}^{*}\right)=\min _{\boldsymbol{\theta}} \mathbf{J}(\boldsymbol{\theta}), \\
& \text { s.t. } \quad M_{s} \leq M_{s, \text { max }}
\end{aligned}
$$




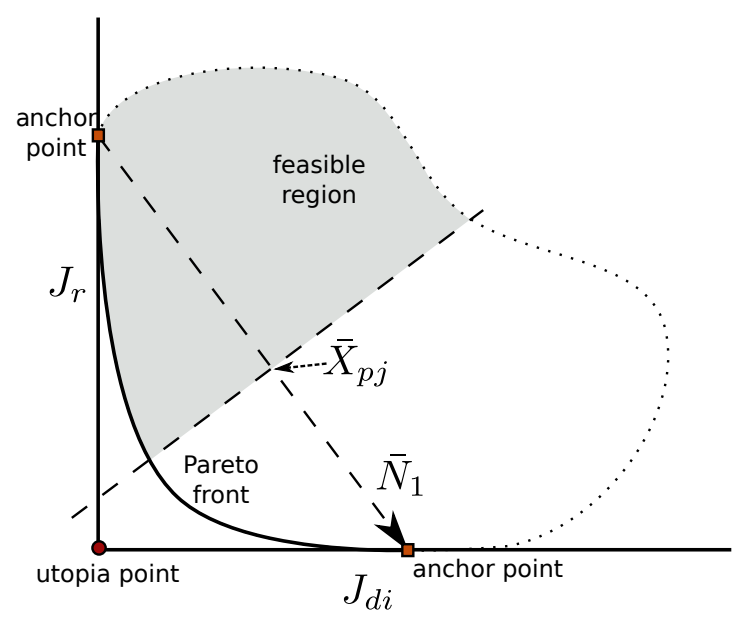

Figure 3. NNC optimization method.

where $M_{s, \max }$ is the maximum robustness value allowed. In other words, this problem is equivalent to find the Pareto front for all possible robust 2DoF PID controllers applied to a SOPTD plant taking into account servo and regulatory modes.

\section{The ENNC Method}

The ENNC, is a new formulation of the original NNC method [14] for multiobjective optimization problems.

The main idea of the NNC methodology is presented in Fig. 3: the utopia plane, which is the plane that joins the anchor points ${ }^{1}$ is parametrized using two variables $\alpha_{1 N N C}$ and $\alpha_{2 N N C}$. Each point $\overline{\mathbf{X}}_{p j}$ along the utopia plane, is computed as:

$$
\overline{\mathbf{X}}_{p j}=\alpha_{1 N N C} \hat{\mathbf{J}}\left(\theta_{1}^{*}\right)+\alpha_{2 N N C} \hat{\mathbf{J}}\left(\theta_{2}^{*}\right) .
$$

with $\alpha_{1 N N C}+\alpha_{2 N N C}=1$ and $\hat{\mathbf{J}}$ the normalized version of $\mathbf{J}$ where, for each component $i$ :

$$
\hat{\mathbf{J}}^{i}=\frac{\mathbf{J}^{i}-\min \left(\mathbf{J}^{i}\right)}{\max \left(\mathbf{J}^{i}\right)-\min \left(\mathbf{J}^{i}\right)}
$$

Then, the original feasible region is constrained using a normal plane to the utopia plane that passes through $\overline{\mathbf{X}}_{p j}$. With this new feasible region, it is only required to minimize one of the functions (e.g. $J_{r}$ ) in order to find the Pareto front. By varying $\overline{\mathbf{X}}_{p j}$, the Pareto front can be obtained solving the optimization problem:

$$
\begin{gathered}
\min _{\theta} \hat{J}_{d o}(\theta), \\
\text { s.t. } \overline{\mathbf{N}}_{1}^{T}\left(\hat{\mathbf{J}}(\theta)-\overline{\mathbf{X}}_{p j}\right) \leq 0, \\
h(\theta)=0, \\
g(\theta) \leq 0,
\end{gathered}
$$

where $\overline{\mathbf{N}}_{1}$ is the vector that contains the direction of the utopia plane. The NNC method however, supposes that for that each anchor point contains only one of the minimal value of each function and one of the maximum value. This

\footnotetext{
${ }^{1}$ in the case of minimizing only two functions, the utopia plane reduces to a line
}

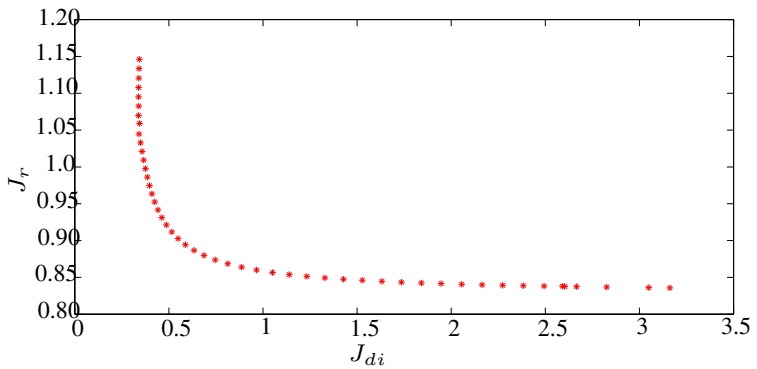

Figure 4. The Pareto frontier for the benchmark process

is always true for the case of minimizing two functions, however is not necessarily true when minimizing three or more functions. For this reason, the ENNC proposes a redefinition of the anchor points and a linear transformation between the objective functions in order to obtained the correct scale to improve the even distribution of the Pareto front when applying the NNC [15].

Due to page constraint, a deeper review of the ENNC method cannot be presented in this paper. However, the interested reader can follow [15] and references therein for a complete presentation of the method. The proposed steps in this paper, in order to solve (12) can be summarized as follows:

1) Define the parameters of the process according to (1).

2) Define $M_{s, \max }$ to select the desired level of robustness.

3) Apply the ENNC method in order to find all the needed points of the Pareto-front.

4) Once the Pareto front has been obtained, proceed to select the more appropriate tuning to the problem at hand.

\section{RESULTS}

\section{A. Comparison of different PID tuning for a benchmark problem}

In order to test the capabilities of a 2DoF-PID controller optimized using the ENNC method, a benchmark process presented in [16] is used for comparison. It is a fourth order plant given by:

$$
P(s)=\frac{1}{\prod_{n=0}^{n=3}\left(0.5^{n} s+1\right)} .
$$

A low order model can be found in order to design a suitable PID controller for the full order plant. Using the $123 \mathrm{c}$ method [17], the obtained model is given by:

$$
F(s)=\frac{e^{-0.297 s}}{(0.9477 s+1)(0.6346 s+1)}
$$

In Figure 4, the Pareto frontier obtained for the model (16) is presented, the curve was obtained with the ENNC optimization of the cost functions $J_{r}$ and $J_{d i}$, and the optimal parameters of PID controller with two degrees of freedom were obtained.

It is interesting to note that, in order to improve the response of $J_{d i}, J_{r}$ has to be augmented (worsening the regulator response), however the degradation is not as much as the improvement in the $J_{d}$ function. This is a clear example of one 


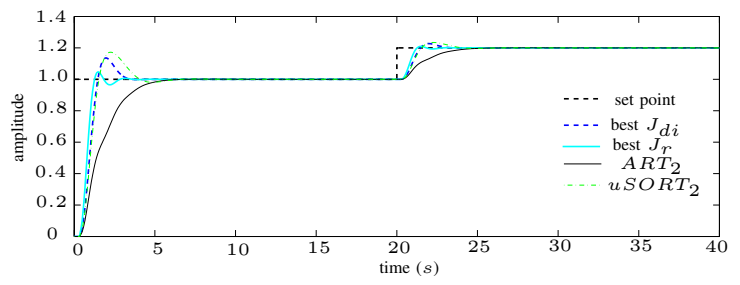

Figure 5. Optimal response of the control system $J_{r}$

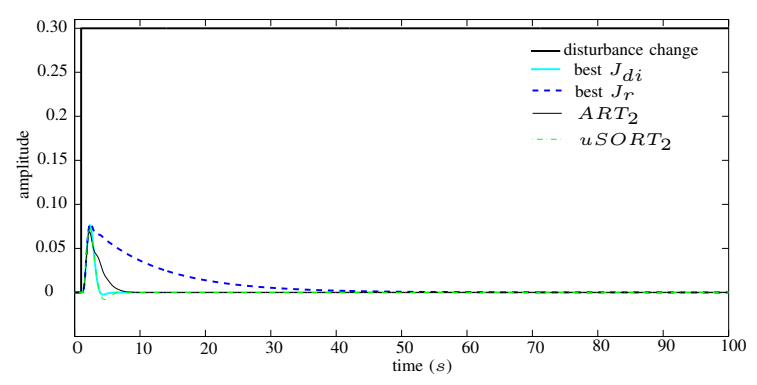

Figure 6. Optimal response of the control system $J_{d i}$

of the many advantages of using a multi-objective framework for controller tuning.

The optimal parameters for the 2Dof-PID controller, for optimum tuning in disturbance rejection $\left(J_{d i}\right)$ and optimal servo control $\left(J_{r}\right)$, are presented in in Table I, along with two other tunings: the $\mathrm{ART}_{2}$ method [18] and the uSORT 2 [19], to compare the performance of the closed loop control. It is important to clarify that these two tuning are just the extreme points of the Pareto front, thanks to the ENNC method, the control engineer is able to select any closed-loop response between these two extremes, all of them equally optimal and robust.

In Fig. 5, the response of the control loop with the four different tunings is presented, for a change in the reference value. The best system response is given by the ENNC optimal controller $J_{r}$, emphasizing its characteristics of less IAE, as shown in Table II.

The optimal controller for disturbance rejection is presented in Fig. 6. Also it is clear how the loop response with optimal controller for changes in reference value is the worst for disturbance rejection.

\section{B. 2DoF-PID optimized tuning for a non-linear CSRT process}

Continuous stirred tanks reactors (CSTR) are one of the most common sub-system in the chemical process field. Depending on the reaction, the dynamic of this plant can be

Table I

PID CONTROLLER PARAMETERS USING TWO DEGREES OF FREEDOM.

\begin{tabular}{ccccc}
\hline Tuning & $K_{c}$ & $T_{i}$ & $T_{d}$ & $\beta$ \\
\hline optimum $J_{d i}$ & 3.3750 & 1.0812 & 0.3095 & 0.5466 \\
optimum $J_{r}$ & 3.0572 & 8.4419 & 0.3986 & 1.2329 \\
$A R T_{2}$ & 3.3657 & 1.7636 & 0.4884 & 0.2971 \\
$u S O R T_{2}$ & 3.1708 & 0.8997 & 0.3945 & 0.4731 \\
\hline
\end{tabular}

Table II

SERVO PERFORMANCE RESPONSE

\begin{tabular}{ccc}
\hline Tuning & IAE & $M_{s}$ \\
\hline optimum $J_{r}$ & 1.004 & 2 \\
optimum $J_{d i}$ & 1.297 & 2 \\
$u S O R T_{2}$ & 1.522 & 2 \\
$A R T_{2}$ & 2.121 & 2 \\
\hline
\end{tabular}

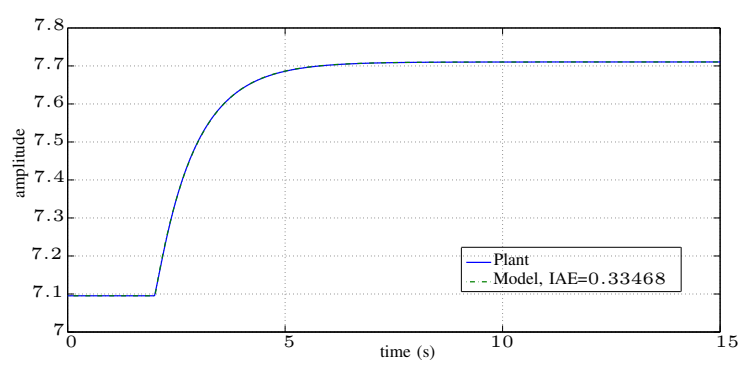

Figure 7. Comparison between the nonlinear response of the CSTR and the linear model.

highly non-linear, however, it is common to operate them in a given operation point with the controllers tuned for disturbances rejection.

Consider the CSTR non-linear model based on [20] and references therein:

$$
\begin{aligned}
\dot{x}_{1} & =-x_{1}+D_{a}\left(1-x_{1}\right) e^{\frac{x_{2}}{1+x_{2} / \varphi}}, \\
\dot{x}_{2} & =-(1+\delta) x_{2}+B D_{a}\left(1-x_{1}\right) e^{\frac{x_{2}}{1+x_{2} / \varphi}}+\delta u, \\
y & =x_{2},
\end{aligned}
$$

where $x_{1}$ and $x_{2}$ represents dimensionless reactant concentration and reactor temperature, $u$ is the dimensionless cooling jacket temperature which is considered as the control input, $y$ is the output of the system, $D_{a}=0.072$ is the Damköhler number, $\varphi=20$ is the activated energy, $B=8$ is the heat of the reaction and $\delta=0.05$ is the heat transfer coefficient. The system is considered to be controlled from the equilibrium point $x_{1}=0.931$ and $x_{2}=7.095$ with input $u=0$.

In order to find a suitable PID controller for this plant, it is necessary to find a low order linear model. After performing a step change of amplitude $\Delta u=10$ in the input, the model found is given by:

$$
F(s)=\frac{0.061 e^{-0.0037}}{(0.9213 s+1)(0.0056 s+1)} .
$$

This model fits the plant dynamics at the operating point very well, as can be seen in Fig. 7, where the integral of the absolute error (IAE) between the output of the plant and the output of the model is 0.33468 .

In Fig. 8, the Pareto frontier obtained using the CSTR model is showed. Again, the curve was obtained with the ENNC optimization method with the cost functions $J_{r}$ and $J_{d i}$.

The tuning parameters for a 2DoF-PID controller optimized for this process, are presented in Table III. The response obtained for an optimum tuning for changes in the reference value is showed in Fig. 9, while the obtained performance 


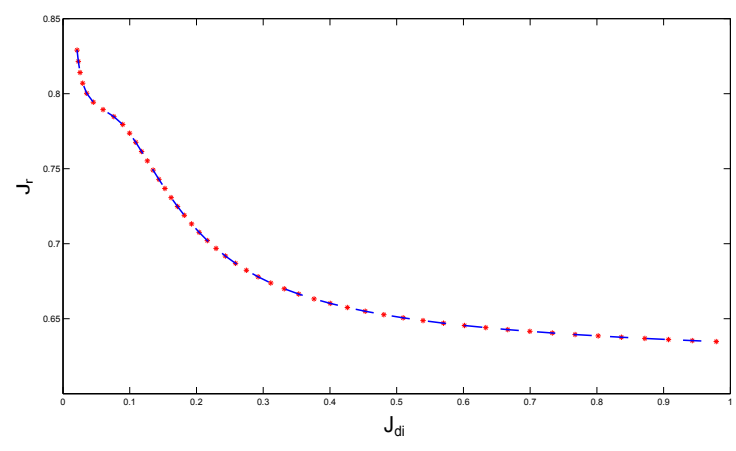

Figure 8. The Pareto frontier for the CSTR process

Table III

2DOF PID CONTROLLER, FOR THE CSRT.

\begin{tabular}{ccccc}
\hline Tuning & $K_{p}$ & $T_{i}$ & $T_{d}$ & $\beta$ \\
\hline optimum $J_{d i}$ & 10 & 0.1 & 0.1 & 1.9406 \\
optimum $J_{r}$ & 10 & 10 & 0.1 & 2.5759 \\
\hline
\end{tabular}

values are presented in Table IV. While the tuning was computed using the linear low order model, the simulation presented in this paper was performed using the non-linear model of the CSTR. It is clear how the performance of the best $J_{r}$ tune overcomes the closed loop response of the best $J_{d i}$ tune, presenting a minor IAE index.

In Table $\mathrm{V}$, the corresponding values of IAE for the servo and regulator responses are presented. Note the differences between $J_{d i}$ and $J_{r}$ in tables IV and V. The obtained Pareto front shows that considerable changes in $J_{d i}$ does not correspond to large degradation in $J_{r}$. This is consistent with the simulation results shown, and therefore, one can initially tune the controller for optimal $J_{d i}$, knowing that the servo response is not highly degraded, while also guaranteeing a safe robustness value. If a better servo response is needed,

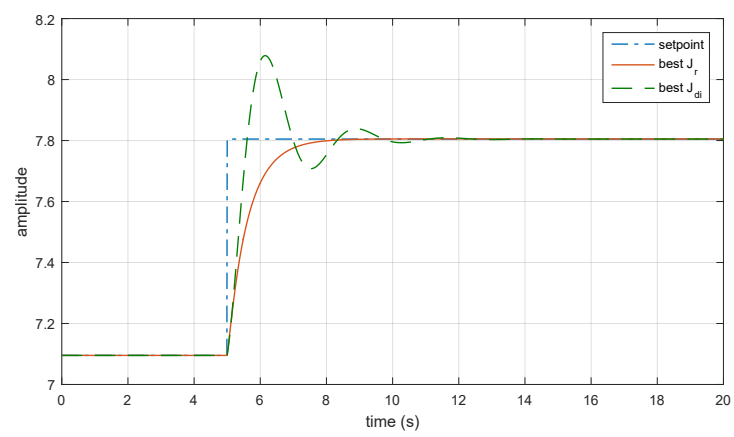

Figure 9. Setpoint tracking for the CSRT system.

Table IV

PERFORMANCE RATES WITH OPTIMAL TUNING $J_{r}$, FOR THE CSRT.

\begin{tabular}{ccc}
\hline Tuning & IAE & $M_{s}$ \\
\hline optimum $J_{r}$ & 0.4547 & 2 \\
optimum $J_{d i}$ & 0.5835 & 2 \\
\hline
\end{tabular}

Table V

Performance Rates With optimal tuning $J_{d i}$, FOR THE CSRT.

\begin{tabular}{ccc}
\hline Tuning & IAE & $M_{s}$ \\
\hline optimum $J_{r}$ & 4.9390 & 2 \\
optimum $J_{d i}$ & 0.1108 & 2 \\
\hline
\end{tabular}

it can be found by picking other tuning that also belongs to the Pareto front, but with the knowledge on how this decision affects the regulator response.

\section{Optimization considering three sources of disturbances}

One of the advantages of the ENNC method is that it can tackle more than two cost functions using the same formulation of the problem. Other methods like the LCR performs better than the NNC and NBI [21], but are suited only for two function problems.

Consider Fig. 1 where $r(s), d_{i}(s)$ and $d_{o}(s)$ are sources of disturbances to the plant, since a change in any of these signals drives a change in the output $y(s)$. Using the same multi-objective framework, it is possible to find all the Paretooptimal controllers that involve any of these three disturbances.

If $J_{d o}(\boldsymbol{\theta})$ is defined as:

$$
J_{d o}(\boldsymbol{\theta})=\int_{0}^{\infty}\left|-y_{d o}(t, \boldsymbol{\theta})\right| d t,
$$

The composite cost function is given by:

$$
\boldsymbol{J}_{3}(\boldsymbol{\theta})=\left[\begin{array}{lll}
J_{d i}(\boldsymbol{\theta}) & J_{d o}(\boldsymbol{\theta}) & J_{r}(\boldsymbol{\theta})
\end{array}\right]^{T} .
$$

There is only one detail that has to be taken into account when considering the function $\boldsymbol{J}_{3}(\boldsymbol{\theta})$. It is clear that $J_{d i}(\boldsymbol{\theta})$ and $J_{d o}(\boldsymbol{\theta})$ do not depend on $\beta$, therefore, the anchor points where these two functions are minimized are not unique, because for any given $\beta$, the same minimum for $J_{d i}(\boldsymbol{\theta})$ and $J_{d o}(\boldsymbol{\theta})$ is achieved. Considering this, in order to apply the ENNC to the three functions problem, the anchor point are define as follows:

- The value of $\beta$ for the anchor point where $J_{d i}(\boldsymbol{\theta})$ is minimum, is found by minimizing $J_{r}(\theta)$, but fixing the values of $K_{p}, T_{i}$ and $T_{d}$ that minimize $J_{d i}(\boldsymbol{\theta})$.

- The value of $\beta$ for the anchor point where $J_{d o}(\boldsymbol{\theta})$ is minimum, is found by minimizing $J_{r}(\theta)$, but fixing the values of $K_{p}, T_{i}$ and $T_{d}$ that minimize $J_{d o}(\boldsymbol{\theta})$.

- The anchor point where the minimum of $J_{r}(\boldsymbol{\theta})$ is found, is computed as usual.

To simplify the analysis, the model of the controlled process and the controller parameters are normalized [6]:

$$
\hat{s}=T s, \tau_{0}=\frac{L}{T}, \tau_{i}=\frac{T_{i}}{T}, \tau_{d}=\frac{T_{d}}{T}, \kappa_{p}=K_{p} K .
$$

Then the normalized parameters of the controller are, $\hat{\boldsymbol{\theta}}=$ $\left[\kappa, \tau_{i}, \tau_{d}, \beta\right]^{T}$.

Using this normalization, it is possible to find the optimal controllers for a complete set of plants. If for example the parameters of the normalized plant are $\kappa_{p}=1, \tau_{0}=0.5$ and 


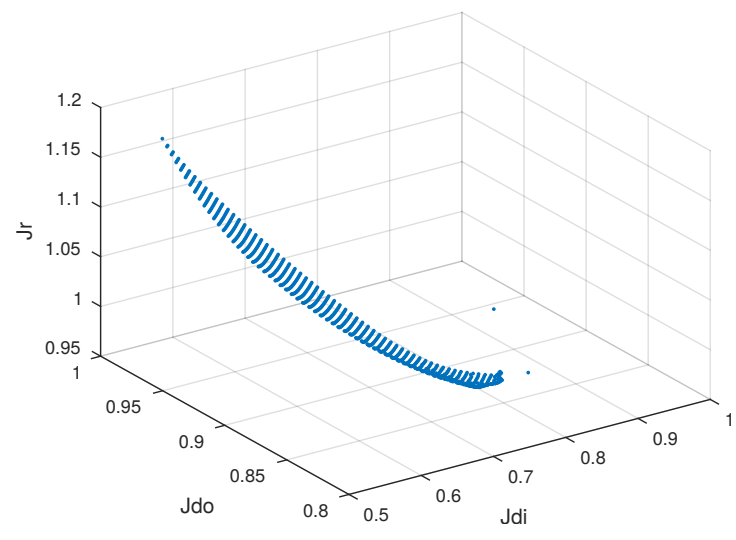

Figure 10. Pareto front obtained using the ENNC method with three sources of disturbances.

$a=0.1$, the Pareto front found is presented in Fig 10. One of the main reasons why the front is narrow, is because the feasible region is constraint by the $M_{s}=2.0$ criterion. This particular Pareto contains 1042 possible controller tunings, all of them optimal in the Pareto sense.

For this work, more than 500 different normalized plants were considered. On average, for each plant, a Pareto of 1000 points was found, yielding near 500000 different tuning parameter, all optimal in the Pareto sense. With that data base, two different options can be follow: in one hand, it is possible to fit a tuning rule based on the data, and in the other hand, a program can be written in order to interpolate a set of parameters, based on the database and parameters of the plant. Both approaches are going to be presented elsewhere.

\section{CONCLUSIONS}

In this paper, the ENNC method was successfully applied to find the optimal parameters of a 2DoF PID controller with robustness constraints.

The method was also applied to a high order model and to a non-linear CSTR plant with excellent results. The results of the tuning were compared with other optimal/robust PID tuning methodologies.

Finally, the tuning problem was augmented in order to handle three different sources of disturbances (change in the reference signal, change in the input disturbance signal and change in the output disturbance signal). The methodology was well suited for this problem and in fact, a database comprising nearly 500000 different controller tuning was found.

\section{ACKNOWLEDGMENTS}

This work was done with grant 322-B4-218 by Vicerrectoría de Investigación de la Universidad de Costa Rica. The code for the ENNC implementation was based on the work by Filip Logist from the Department of Chemical Engineering at Katholieke Universiteit Leuven, Belgium.

\section{REFERENCES}

[1] O. Garpinger, T. Hägglund, and K. J. Åström, "Criteria and trade-offs in PID design," in Proceedings of the IFAC Conference on Advances in PID Control, Brescia, Italy, March 28-30 2012.
[2] O. Arrieta and R. Vilanova, "PID Autotuning settings for balanced Servo/Regulation operation," in 15th IEEE Mediterranean Conference on Control and Automation (MED07), June 27-29, Athens-Greece, 2007.

[3] — "Performance degradation analysis of controller tuning modes: Application to an optimal PID tuning," International Journal of Innovative Computing, Information and Control, vol. 6, no. 10, pp. 4719-4729, 2010.

[4] A. Gambier, "Optimal PID controller design using multiobjective Normal Boundary Intersection technique," in Asian Control Conference, 2009. ASCC 2009. 7th, Hong Kong, China, 27-29 August 2009 2009, pp. $1369-1374$.

[5] S. Tavakoli, I. Griffin, and P. Fleming, "Robust pi controller for load disturbance rejection and setpoint regulation," in Control Applications, 2005. CCA 2005. Proceedings of 2005 IEEE Conference on, aug. 2005, pp. $1015-1020$.

[6] J. Rojas, D. Valverde-Mendez, and V. Alfaro, "CACSD tool for optimal multi-objective PI controller tuning," in Intelligent Engineering Systems (INES), 2013 IEEE 17th International Conference on, June 2013, pp. 107-112.

[7] H. S. Sánchez and R. Vilanova, "Multiobjective tuning of PI controller using the NNC method: Simplified problem definition and guidelines for decision making," in 2013 IEEE 18th Conference on Emerging Technologies Factory Automation (ETFA), 8 2013, pp. 1-8.

[8] A. Bagis, "Tabu search algorithm based PID controller tuning for desired system specifications," Journal of the Franklin Institute, vol. 348, no. 10 , pp. $2795-2812,2011$.

[9] I. Chiha, N. Liouane, and P. Borne, "Tuning PID controller using multiobjective ant colony optimization," Applied Computational Intelligence and Soft Computing, vol. 2012, pp. 1-7, 2012, article ID 536326.

[10] Q. Li, M. Liu, and H. Liu, "Piecewise normalized normal constraint method applied to minimization of voltage deviation and active power loss in an ac-dc hybrid power system," IEEE Transactions on Power Systems, vol. 30, no. 3, pp. 1243-1251, May 2015.

[11] O. Arrieta and R. Vilanova, "Simple PID tuning rules with guaranteed $M_{s}$ robustness achievement," in 18th IFAC World Congress, August 28September 2, Milano-Italy, 2011.

[12] _ "Simple Servo/Regulation Proportional-Integral-Derivative (PID) Tuning Rules for Arbitrary $M_{s}$-Based Robustness Achievement," Indus trial \& Engineering Chemistry Research, 2012, dOI: 10.1021/ie201655c.

[13] R. Marler and J. Arora, Survey of multi-objective optimization methods for engineering, Structural and Multidisciplinary Optimization, vol. 26, pp. 369-395, Optimal Design Laboratory, College of Engineering, The University of Iowa,USA, febrero 2004.

[14] A. Messac, A. Ismail-Yahaya, and C. Mattson, "The normalized normal constraint method for generating the Pareto frontier," Structural and Multidisciplinary Optimization, vol. 25, pp. 86-98, 2003.

[15] J. Sanchis, M. Martínez, X. Blasco, and J. Salcedo, "A new perspective on multiobjective optimization by enhanced normalized normal constraint method," Structural and Multidisciplinary Optimization, vol. 36, no. 5, pp. 537-546, 2008. [Online]. Available: http: //dx.doi.org/10.1007/s00158-007-0185-4

[16] K. J. Åström and T. Hägglund, "Benchmark systems for PID control," in Proceedings IFAC Workshop Digital Control: Past, Present and Future of PID Control, 2000, 5-7 abril, Terrasa, España.

[17] V. M. Alfaro, "Identificación de modelos de orden reducido a partir de la curva de reacción del proceso," Ciencia y Tecnología, vol. 24, no. 2, pp. 197-216, 2006, (in Spanish). [Online]. Available: http: //revistas.ucr.ac.cr/index.php/cienciaytecnologia/article/view/2647/2598

[18] R. Vilanova, V. M. Alfaro, and O. Arrieta, "Analytical robust tuning approach for two-degree-of-freedom PI/PID controllers," Engineering Letters (IAENG), vol. 19, pp. 204-214, 2011.

[19] V. M. Alfaro and R. Vilanova, Optimal Robust Tuning for 1DoF PI/PID Control Unifying FOPDT/SOPDT Models, IFAC Conference on Advances in PID Control, Brescia, Italy, Marzo 2012.

[20] W.-D. Chang, "Nonlinear CSTR control system design using an artificial bee colony algorithm," Simulation Modelling Practice and Theory, vol. 31, no. 0, pp. $1-9,2013$.

[21] J. Rojas, D. Valverde-Mendez, V. Alfaro, O. Arrieta, and R. Vilanova, "Comparison of multi-objective optimization methods for pi controllers tuning," in Emerging Technologies Factory Automation (ETFA), 2015 IEEE 20th Conference on, Sept 2015, pp. 1-8. 